\title{
Dynamic Simulation of Bidirectional Low-Temperature Networks - A Case Study to Facilitate the Integration of Renewable Energies
}

\author{
Tobias Blacha ${ }^{1}$, Michael Mans ${ }^{1}$, Peter Remmen ${ }^{1}$, Dirk Müller ${ }^{1}$ \\ ${ }^{1}$ RWTH Aachen University, E.ON Energy Research Center, \\ Institute for Energy Efficient Buildings and Indoor Climate, Germany
}

\begin{abstract}
The global climate targets lead to a growing interest in supplying buildings with heat and cold more efficiently. In districts with simultaneous heating and cooling demands, bidirectional low-temperature networks can be an efficient alternative to balance local demands within one thermal network. In order to examine this concept in more detail, this paper presents an integral dynamic simulation model of a bidirectional low-temperature network with 15 connected buildings, equipped with heat pumps and chillers in Modelica. Performing different simulation studies regarding temperature levels of the system as well as storage utilization and the integration of renewable energy sources, the general benefits and barriers of this innovative concept are evaluated.
\end{abstract}

\section{Introduction}

The integration of renewable energy sources such as solar or geothermal energy and decentralized heat sources will become even more important in the future. In addition, the coupling of the heating, cooling and electricity sectors is also becoming increasingly important in the energy supply of urban districts. Bidirectional low-temperature networks represent a supply concept for districts that allows the integration of various renewable energy technologies due to the low temperature requirements on the one hand and on the other hand implies the coupling of electricity, heat and cold through the operation of decentralized heat pumps and chillers. These networks provide buildings with thermal energy close to the ambient temperature and local heat pumps utilize the fluid with low-temperatures as a heat source to lift the temperature level suitable for the building heating system. At the same time, the networks serves as a sink for waste heat from chillers, refrigeration or heat from other low-temperature sources. According to The European Comission (2015), bidirectional low-temperature networks can be assigned to the fifth generation of district heating networks. The fifth generation of thermal networks differs from the four previous generations described by Lund et al. (2014) in

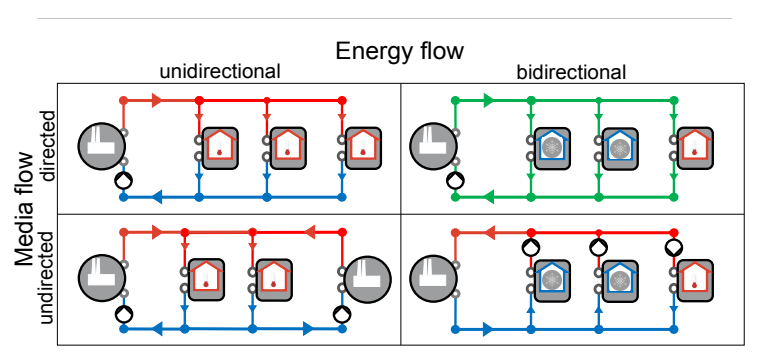

Figure 1: Network typology classification of low-temperature networks according to Lang et al. (2018).

the fact that they are operated at a neutral temperature level (e.g. $\left.15-20^{\circ} \mathrm{C}\right)$. Low-temperature networks, which also include bidirectional low-temperature networks, exist in different variants. A possible criterion for differentiation is the number of pipelines. Here a distinction is made between thermal networks from one to four lines (Sulzer and Hangartner, 2014). A further differentiation criterion also described by Sulzer and Hangartner (2014) is the consideration of the energy and media flow in the respective network. Regarding the energy flow to the connected buildings the classification subdivides into uni- and bidirectional flow. The media flow in the system can be directed (i. e. central pump) and undirected. Figure 1 gives an overview of the different system configurations.

The bidirectional low-temperature network presented in this paper is categorized in the undirected, bidirectional networks, with a slight adaption of the positioning of the pumps, further described in the Methodology section. Nevertheless, all categories have the central balancing unit in common. This unit ensures the system operation within certain boundary temperature conditions. Various conventional and renewable generation technologies can be integrated as balancing units, such as CHPs, central heat pumps, compression and absorption chillers, solar and geothermal as well as connections to central heating and cooling networks. Thus, bidirectional lowtemperature networks are a promising technology for the energy-efficient supply of heat and cold in urban areas. However, there are still challenges that need to be overcome in order to achieve comprehensive use. 
Sulzer and Hangartner (2014) identify a lack of experience in the planning and operation of the networks, as well as a lack of fundamentals for evaluating the potential for districts under local conditions. Bidirectional low-temperature networks are characterized by a complex thermo-hydraulic system behavior, which differs fundamentally from conventional district heating and cooling. To meet the challenges of (1) lack of experience of real operation, (2) suitability of local conditions and (3) complex hydraulic systems, dynamic simulation are one promising solution. Dynamic simulation provides detailed insights in the systems behavior and allows to evaluate different system configurations. This paper presents a dynamic simulation model of a bidirectional low-temperature network of a real district. We use the model to perform a simulation and give detailed insights into the system operation. To this end, the modeling approach and the system model are presented first, followed by the description of the example district. Subsequently, various system configurations are considered using the dynamic simulation model, including the influence of network temperatures and demand structure on system operation. Furthermore, different possibilities for the integration of renewable energies into the energy supply of bidirectional low-temperature networks are discussed.

\section{Methodology}

Using an undirected, bidirectional low-temperature network in the scope of this paper, the description of the overall concept takes place in more detail in this section. As the presented evaluations are performed with a dynamic simulation model, the simulation models are described later in this section.

\section{Network concept and models}

The principals of the presented undirected, bidirectional networks were already introduced above. We divide the network into three major sub-systems, the central balancing unit, the substation in the buildings and the pipe network itself. This section starts with the detailed description of the used substation concept. The undirected, bidirectional networks considered in this paper differs from the conventional network types in the positioning of the distribution pumps, as these are installed decentral in the substations of the buildings. Due to the decentralized distribution of the pumps, it is possible for every consumer to obtain the mass flow required to cover the heating and cooling demands on their own from the network. For this purpose, the buildings are equipped with heat pumps and chillers that utilize the thermal network as an energy source. The buildings are connected to the cold and warm line of the bidirectional network, the warm line serves as a heat source for heat pumps, the cold line serves as a heat sink of chillers. Consequently, the medium with reduced temperature by the evaporator of the heat pump and the heated

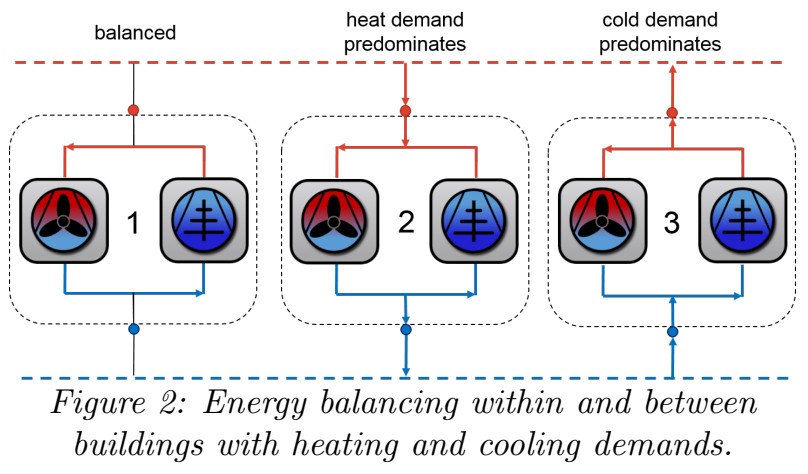

medium of the condenser of the chiller are fed into the cold and warm line of the network respectively. Both, the cold and warm line are operated at low temperatures in order to minimize heat losses to the ambient and to provide good conditions for efficient heating and cooling. Thus these networks enable the user to take heat or cold as required while simultaneously feeding the cold/waste heat into the other line.

By connecting buildings with different thermal demands for both heating and cooling, bidirectional low-temperature networks enable efficient use of thermal energy through energy balancing between different users. The concept is to recycle as much thermal energy as possible within the buildings or to exchange it between different users. Energy balancing within a building takes place with simultaneous heating and cooling demands in the same building, energy balancing between different buildings with heating and cooling demands takes place using the thermal network Figure 2 schematically illustrates these two energy balancing processes within the system. A building is shown on the left (building 1), where heating and cooling demands are completely balanced. In this situation the building is decoupled from the network. The other two buildings have a predominant heat demand (building 2) and a predominant cold demand (building 3), so that they need heat or cold from the network. Since the pumps are installed decentral in the substations of the respective buildings, the flow direction within the network is determined by the composition of the energy demands of the individual buildings. Thus, the flow direction of the medium within the network is regulated according to the demand situation and can change. In order to ensure the network temperature limits, a central balancing unit is used.

The analysis of the bidirectional low-temperature network are carried out using dynamic, thermo-hydraulic simulation models developed in the modeling language Modelica. For this purpose, a system model of the bidirectional network is composed of various component models (Müller et al., 2016), (Wetter et al., 2015). Figure 3 shows the schematic structure of the system model. The three main components of the system model are the models of the hydraulic network (i.e. junctions and pipes), the substations representing the buildings heating and cooling de- 


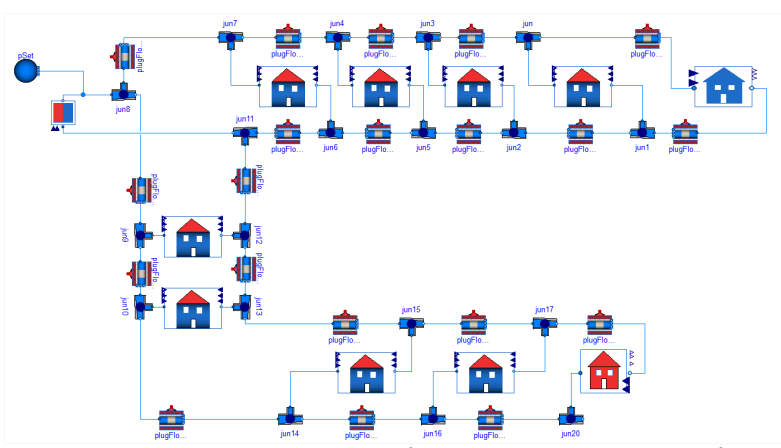

Figure 3: Visualization of the system model of the bidirectional low-temperature network.

mands as well as the building-integrated equipment (heat pumps, chillers and distribution pumps) and the model of the central supply unit. According to the considered network topology, these models are combined to the system model of the respective bidirectional low-temperature network by connecting the individual component models. The pipe elements are modeled using a dynamic, equation-based thermohydraulic pipe model, the so-called plug-flow pipe model (van der Heijde et al., 2017). The model bases on a plug-flow approach, suitable for the simulation of long pipes, e.g. in district heating and cooling systems. Therefore, the pipe model takes into account the transport delay, heat loss and storage effects as well as temperature propagation along the pipe length idealized as plug-flow. The plug-flow pipe model copes with heating and cooling systems, fluctuating inlet temperatures, varying mass-flows (stopping or reversing) and arbitrary network lay-outs including branching and meshed systems. For a more accurate calculation of the heat losses of the pipes, the model was extended to include the surrounding soil, which is represented by a combination of cylindrical thermal resistances $(\mathrm{R})$ and capacitances $(\mathrm{C})$. These $\mathrm{RC}$-combinations represent the heat transfer and heat storage effects in the pipe and the surrounding soil. As an external boundary condition for the heat loss calculation, the annual profile of the undisturbed soil temperature is used, which is calculated according to Florides and Kalogirou (2005).

In the system model, the substation models represent the buildings connected to the network as well as the equipment installed in the substations. This includes not only heat pumps and chillers but also the distribution pumps, which are required to provide the mass flow for heating and cooling applications. The demand profiles are inputs of the substation models, which can either be determined by dynamic building simulations or given by measured values. These profiles are used in the substations for the equation-based control of mass flows. To this end, the required mass flow is calculated on the basis of the current heating or cooling demand by specifying a temperature difference between flow and return and passed on to the pump models. For modeling the heat pumps and chillers in the substations, a power curve adjusted depending on the Carnot efficiency is used. In these models, the coefficient of performance ( $\mathrm{COP}$ ) is calculated as a function of the source and sink temperature using a quality grade of the heat pump or chiller.

The main function of the central supply unit is to ensure that the limits of the network temperatures are not exceeded. Therefore the model contains two heat exchangers for heating and cooling. The direction of flow within the central balancing unit is determined by the buildings and indicates whether the current demand for heat or cold is predominant. In the case of a predominant heat demand, heat must be added to the network; in the case of a predominant cooling demand, heat must be extracted from the network accordingly. As long as the network temperatures are within the defined temperature range, the heat exchangers neither add nor remove heat from the network. The model of the central balancing unit allows to simulate the required heat and cold supply without considering specific equipment for heat and cold generation.

\section{Use case and simulation studies}

The above described integral dynamic simulation model is used for an existing city district composed of 15 buildings connected to the bidirectional network with ten substations. The city district, called Medicon Village, is located in Lund, southern Sweden and consists of a mixture of commercial and residential buildings. In this area, E.ON is currently building a bidirectional low-temperature network under the name ectogrid ${ }^{T M}$ (ectogrid $\left.d^{T M}, 2019\right)$. Figure 3 shows the structure of the bidirectional low-temperature network represented by the system model. Eight substations are connected to buildings with heating and cooling demands, one substation supplies buildings with cold only and one substation supplies buildings with heat only. In addition, a central energy balancing unit is integrated in the system model to ensure compliance with the network's temperature limits. The temperature levels required for heating and cooling the buildings are $55 / 45^{\circ} \mathrm{C}$ and $8 / 14^{\circ} \mathrm{C}$ respectively. The distribution network of the bi-directional low-temperature network has a total pipe length of approx. $3.1 \mathrm{~km}$ and consists of plastic pipes without insulation due to the low temperature difference to the surrounding soil and the resulting low heat losses. The aggregate annual heat consumption of the buildings is about $9.4 \mathrm{GWh}$ with a peak load of $4.0 \mathrm{MW}$. The annual cooling consumption is $3.8 \mathrm{GWh}$ with a peak power of $2.8 \mathrm{MW}$. Annual heat and cold consumption profiles are measured in hourly resolution and are used as simulation inputs. Figure 4 shows the annual aggregated, measured consumption profiles. For the energy efficiency of bidirectional lowtemperature networks, the simultaneous occurrence of heating and cooling demands is particularly important, since in these cases a direct energy balancing 


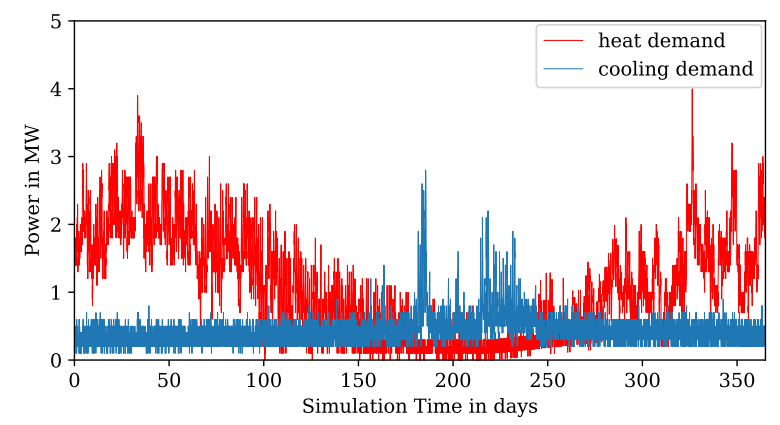

Figure 4: Aggregated heat and cold consumption profiles of Medicon Village.

can take place between buildings. An evaluation of the demand structure is achieved using the diversity index according to Pass et al. (2018). This index can be used to evaluate the energy efficiency of a bidirectional low-temperature network based on the demand structures and the operational energy efficiency of the heat pumps and chillers. For the analyzed use case, the annual average value of the diversity index is 0.74 , which indicates an energy-efficient operation of the bidirectional network.

In the following section, the dynamic simulation model of the example district Medicon Village is used to investigate different system configurations of bidirectional low-temperature networks. The system behavior is first analyzed on the basis of a base case, followed by the investigation of different temperature levels, the investigation of the influence of additional consumers and the integration of central storage systems as well as the influence of pipe network insulation. In these studies, the focus is on the system's energy efficiency and the integration of renewable energies.

\section{Results and discussion}

In this section, different system configurations of bidirectional low-temperature networks are discussed using dynamic simulations of the example district. The system variations examined in more detail are the impact of different temperature levels of the thermal network, the influence of the integration of thermal energy storage and the adjustment of the demand structure through the integration of additional consumer as well as the effect of insulation of the pipe network. Based on these studies, the possibilities of integrating different renewable energy resources into the supply of bidirectional low-temperature networks will then be discussed. First, the general operation of the bidirectional low-temperature network is discussed on the basis of a base case simulation, which will later be used as a basis for comparison and evaluation of the different system variations. In this basic case, the supply temperature limit values of the network are set at $20^{\circ} \mathrm{C}$ and $32{ }^{\circ} \mathrm{C}$. The temperature differences over the substations are $5 \mathrm{~K}$ in both heating and cooling operation. In order to avoid overheating

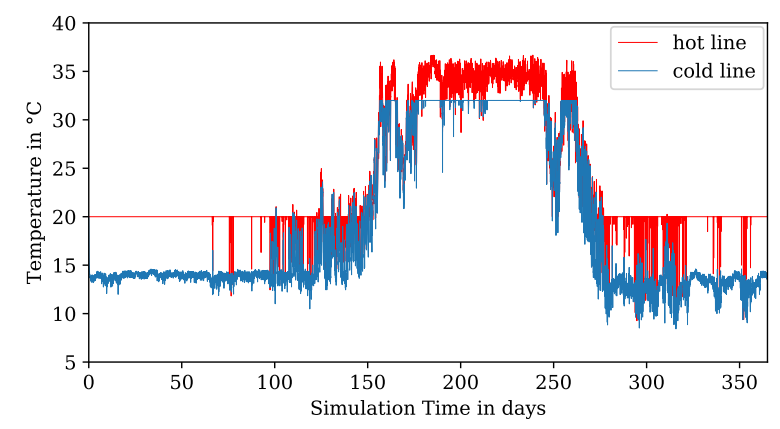

Figure 5: Temperatures of warm and cold line at central supply unit.

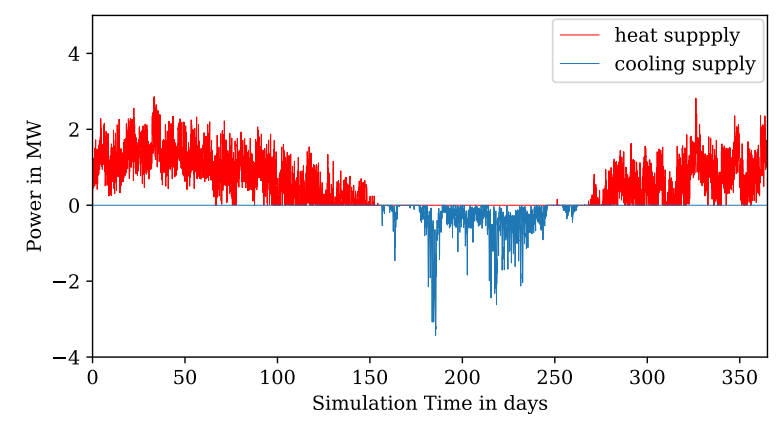

Figure 6: Heat and cold supply of central balancing unit.

and cooling down of the system, the central balancing unit adds or removes heat from the network.

Figure 5 and Figure 6 show the simulation results for the temperature profiles of the warm and cold lines measured at the central supply as well as the heat and cold supply of the central supply unit for the base case. Based on the two diagrams, it can be seen that there is a predominant heat demand in the network in winter, thus heat must be supplied to the network in order to ensure the minimum supply temperature of $20^{\circ} \mathrm{C}$. The return temperatures during this time are slightly below $15^{\circ} \mathrm{C}$ due to the heat extraction of the substations and the heat losses of the pipe network to the surrounding soil. In summer, the network temperature rises due to the increased demand for cooling. In the transition periods between winter and summer the predominant demands alternate, leading to multiple flow reversals in the system and fluctuating temperatures. During this transition periods the energy demands in the network balance each other, the central supply does not have to be operated and the network temperature varies between the limit values. This energy balance between different buildings also lowers the annual amount of heat and cold supplied by the central unit. The annual heat supply of $4.7 \mathrm{GWh}$ is about $50 \%$ of the heat demand, the central cold supply of $0.8 \mathrm{GWh}$ is only about $22 \%$ of the cold demand. In addition, however, a power quantity of $3.6 \mathrm{GWh}$ is required for the operation of the heat pumps and chillers. One main difference to conventional district heating and cooling networks is the simultaneous use of the bidirectional network for heat and cold supply, which is also shown by the cen- 
tral heat and cold supply in Figure 6. Although there are heat and cold demands in the network throughout the year, only the predominant demand has to be balanced by central supply unit.

\section{Temperature levels}

The impact of the different network temperature limits on the energy efficiency is analyzed on the basis of the annual amount of heat and cold supplied by the central balancing unit as well as the electricity demand for the operation of the decentralized heat pumps and chillers. For this purpose, three different temperature limit combinations listed in Table 1 are simulated and compared. Starting from the temperature limits in the base case of $20 / 32^{\circ} \mathrm{C}$, a case with a larger spread between the limit values $\left(16 / 36^{\circ} \mathrm{C}\right)$ and a case with a smaller spread between the limit values $\left(24 / 28^{\circ} \mathrm{C}\right)$ is considered. As Table 1 shows, the network temperatures primarily influence the central heat supply and the power demands of the decentralized equipment. In general, it can be seen that a larger temperature spread leads to a lower need for heat supply, but at the same time to higher power demands of the heat pumps and chillers. The reduction in electricity demand at smaller temperature spreads primarily results from the improved operating conditions for heat pumps and chillers. There are two main reasons for the increase in heat demand for smaller temperature spreads. On the one hand, the higher network temperatures lead to higher heat losses of the pipe network. On the other hand, the improved operating conditions for heat pumps, i.e. the higher COPs, mean that less electricity is required for the heat supply. As a result, more heat has to be extracted from the network to provide the same amount of heat. The cold supply, however, is influenced only to a minor extent by the different temperature levels. First, the heat losses of the network decrease in summer with lower network temperatures; second, the lower network temperature results in improved operating conditions for the chillers and thus to a lower electricity demand. The reduction in heat losses in summer and the lower amount of heat fed into the network due to the improved operation of the chillers are of the same order of magnitude and compensate each other.

In general, this simulation study has shown that the adaptation of the system temperatures of the bidirectional network leads to different amounts of heat and cold supplied by the central balancing unit. Within certain limits, the system can therefore be suited to local conditions and locally available renewable energy resources (e.g. geothermal energy, waste heat) by adjusting the temperature levels. Furthermore, dynamic adaptation of the network temperatures depending on the individual demand situation can lead to an improvement in the energy efficiency of the system. In this context, dynamic simulation models can be used to improve the network temperatures taking local boundary conditions into account.

\section{Additional consumer}

Particularly important, as already described, is the temporal composition of the heating and cooling demands, since the energy efficiency of bidirectional low-temperature networks is strongly determined by the energy compensation between different buildings. Due to the high diversity index of 0.74 , the example district Medicon Village already shows a high potential, but in order to illustrate the influence of the demand structure, the demand structure is adjusted by additional consumers. Especially the simultaneity of heating and cooling demands will be increased leading to a higher diversity index of the bidirectional network. For this purpose, an additional cooling consumer with a constant demand of $200 \mathrm{~kW}$ is connected to the network in winter when the demand for heat predominates, and an additional heat consumer with a constant demand of also $200 \mathrm{~kW}$ is connected in summer when the demand for cold predominates. In the context of urban districts possible additional heating and cooling consumers are data center, super markets and cold storage rooms as well as swimming baths.

The dynamic simulations are performed for the base case with the temperature limits of $20^{\circ} \mathrm{C}$ and $32^{\circ} \mathrm{C}$. Due to the additional consumers, the annual heat demand in the district increases by approx. $6 \%$ to 10.0 GWh and the annual cooling demand by approx. $37 \%$ to $5.2 \mathrm{GWh}$. At the same time the diversity index increases to 0.87 . Although the overall heating and cooling demands of the buildings are higher, the central heat supply is reduced by approx. $30 \%$ to $3.3 \mathrm{GWh}$ and the cold supply by approx. $22 \%$ to $0.7 \mathrm{GWh}$ compared to the base case without additional consumers. At the same time, the electricity demand increases by $10 \%$ to $4 \mathrm{GWh}$ due to the operation of the additional heat pump and chiller. Thus, by planning and optimizing the demand structures, the energy balancing within the network and the energy efficiency of bidirectional low-temperature networks can be improved.

\section{Integration of thermal energy storage}

The next system configuration examined is the integration of thermal energy storages into the bidirectional low-temperature network. Thermal storage systems offers an opportunity to temporally decouple energy supply and demand and thus improve the potential for using fluctuating renewable energies. To this end, the model of the central supply unit is extended by a simple model of a stratified storage tank. The storage tank is directly integrated into the bidirectional network and thus increases the volume and thermal storage capacity of the network. To show the effect of thermal storage systems, simulations with two different storage sizes are carried out and com- 
Table 1: Impact of the network temperature limits on energy demand.

\begin{tabular}{|l|c|c|c|}
\hline $\mathbf{T}_{\min } / \mathbf{T}_{\max }$ & Heat supply & Cooling supply & Electricity demand \\
\hline $16^{\circ} \mathrm{C} / 36^{\circ} \mathrm{C}$ & $4.3 \mathrm{GWh}$ & $0.9 \mathrm{GWh}$ & $4.7 \mathrm{GWh}$ \\
\hline $20^{\circ} \mathrm{C} / 32^{\circ} \mathrm{C}$ (base case) & $4.7 \mathrm{GWh}$ & $0.9 \mathrm{GWh}$ & $3.6 \mathrm{GWh}$ \\
\hline $24^{\circ} \mathrm{C} / 28^{\circ} \mathrm{C}$ & $5.4 \mathrm{GWh}$ & $0.8 \mathrm{GWh}$ & $3.1 \mathrm{GWh}$ \\
\hline
\end{tabular}

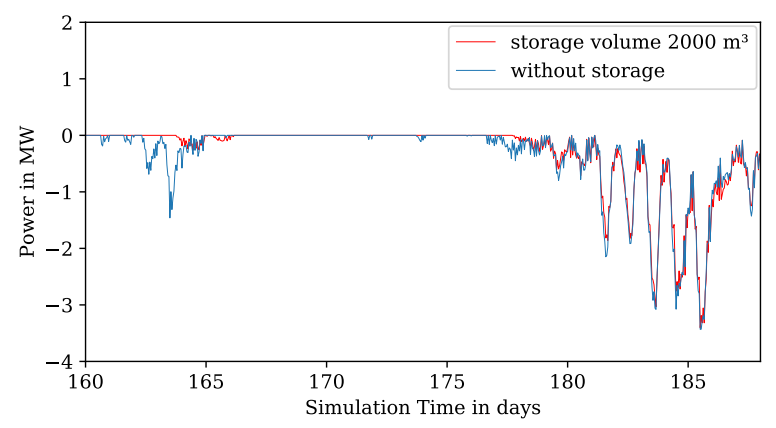

Figure 7: Heat and cold supply of central balancing unit with and without thermal buffer storage.

pared with the base case without storage. The considered storage capacities are $2000 \mathrm{~m}^{3}$ and $200,000 \mathrm{~m}^{3}$ respectively, which corresponds to the cases of a larger buffer storage and a seasonal storage. Because of its size, the seasonal storage in particular is an extreme case which is considered in order to demonstrate the general potential.

First, the influence of the buffer storage on system behavior is discussed. This is most evident in the transition periods in which the predominant heating and cooling demands alternate and the network temperature varies between the limit values without operation of the central supply unit. Figure 7 shows the influence of the buffer storage tank on the central heat and cold supply in the transition time. Due to the higher thermal capacity, the heating-up and cooling-down of the network is less rapid so that the network temperature reaches the limit values more slowly with changing predominant demand. On the one hand, more energy can be stored in the network, and on the other hand, this extends the periods in which the central supply unit does not have to be operated (e.g. between the days 160 to 164). Overall, the central heat and cold supply is slightly reduced, as the additional storage capacity improves the possibilities for energy balancing by allowing a longer period of time between thermal energy feed-in and use. However, the buffer storage has comparatively little effect on the overall systems performance.

The seasonal storage has a much greater influence on system operation. For dimensioning the seasonal storage tank, the cooling demand is used. As can be seen in Figure 6 and Table 1, the annual cooling demand of the bidirectional low-temperature network is significantly lower than the heat demand. The cold resulting from the supply of heat in winter is thus sufficient to cover the entire cooling demand in summer. In order to ensure a realistic initialization of the storage facility, the simulation period is extended to 2 years and the results of the second year are con-

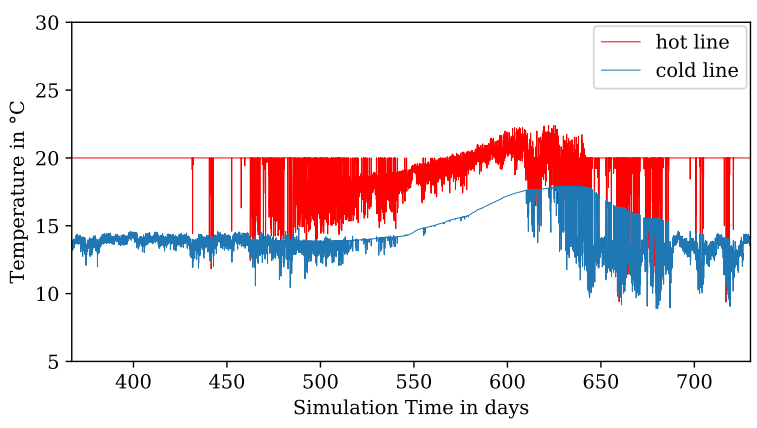

Figure 8: Network temperatures with seasonal thermal storage.

sidered. Figure 8 shows the temperature profiles of the warm and cold lines that occur due to the use of the seasonal storage tank. In winter the system temperatures behave similar to the case without storage shown in Figure 5. In summer, on the other hand, the high thermal capacity of the storage tank leads to very slow heating-up of the network (between the days 530 to 630 ). Despite the high waste heat in summer, the temperature of the warm line only rises slightly to approx. $22^{\circ} \mathrm{C}$ and thus does not reach the limit value. For this reason, the use of the seasonal storage tank allows to supply the example district only with heat and electricity. The central cold supply is no longer required. In addition, the heat supply is reduced by approx. $21 \%$ compared to the base case due to the improved energy balance of the buildings. The electricity demand also decreases by approx. $8 \%$, mainly due to the better operating conditions of the chillers in summer.

\section{Pipe insulation}

Since the insulation of the pipe network has a large impact on the investments of the bidirectional lowtemperature network, the dynamic simulation model can be used to estimate the influence and need of the insulation with regard to critical network temperatures and heat losses. In order to estimate the influence of pipe insulation on the system behavior, a simulation of the base case with insulated pipe network is carried out and examined in the following. An insulation layer is considered in the plug-flow pipe model and parameterized for all pipe sections with a thermal conductivity of $0.04 \mathrm{~W} /(\mathrm{mK})$ and a thickness of the pipe insulation of $4.5 \mathrm{~cm}$. Due to the insulation of the pipe network, the central heat supply is reduced by approx. $16 \%$ to $4.0 \mathrm{GWh}$, but in parallel the central cold supply increases by almost $60 \%$ to approx. 1.4 GWh. The total power demand of the decentralized heat pumps and chillers is almost unaffected. Overall, the pipe insulation leads to a reduction in the heat losses of the network. This reduction 
has a negative effect in winter, when the heat demand in the network predominates and heat has to be supplied to the network. In summer the heat losses of the network have a positive effect, because there is a predominant cooling demand during this period and heat must be extracted from the network. Therefore, the pipe insulation during summer leads to a reduction in heat losses and thus more heat has to be extracted by the central balancing unit. This results in the increase of the annual cold supply. Table 2 summarizes changes in the overall energy demand of the system caused by different pipe insulation levels.

Whether the insulation of the pipe network is advantageous depends strongly on the demand structure, the local conditions (in particular the soil temperature) and energy resources of a district. For example, if cooling can be supplied in a district using renewable energy sources, the increase in cooling demand and the simultaneous reduction in heat demand due to the insulation of the pipe network can lead to reduced $\mathrm{CO}_{2}$ emissions. However, the pipe insulation makes it possible to allow lower network temperatures in winter, as the risk of individual pipe sections freezing due to the medium's stagnation is reduced. The dynamic simulation model can thus help to identify the critical pipe segments that are threatened by freezing, so that the insulation of critical network sections can be taken into account at the planning stage. It allows to test different insulation standards and their impact on the overall energy balance of the system. The simulation results could also serve as a basis for the economic evaluation of the pipe insulation.

Table 2: Impact of pipe insulation level on energy demand.

\begin{tabular}{|l|l|l|l|}
\hline $\begin{array}{l}\text { Insulation } \\
\text { thickness }\end{array}$ & $\begin{array}{l}\text { Heat } \\
\text { supply }\end{array}$ & $\begin{array}{l}\text { Cooling } \\
\text { supply }\end{array}$ & $\begin{array}{l}\text { Electricity } \\
\text { demand }\end{array}$ \\
\hline no insulation & $4.7 \mathrm{GWh}$ & $0.9 \mathrm{GWh}$ & $3.6 \mathrm{GWh}$ \\
\hline $4.5 \mathrm{~cm}$ & $4.0 \mathrm{GWh}$ & $1.4 \mathrm{GWh}$ & $3.6 \mathrm{GWh}$ \\
\hline $10.0 \mathrm{~cm}$ & $3.9 \mathrm{GWh}$ & $1.5 \mathrm{GWh}$ & $3.6 \mathrm{GWh}$ \\
\hline
\end{tabular}

\section{Integration of renewable energies}

The central challenge of the energy sector is the conversion from a predominantly fossil energy supply to a continuously increasing energy supply using renewable energy sources. District heating and cooling systems offer various options for the integration of renewable energies. These are on the one hand the use of renewable energy sources for heat and cold supply, on the other hand the different system components offer possibilities to adapt the energy demand to renewable energy generation. Here, the thermal inertia of buildings, decentralized and central energy storage systems and the thermal inertia of the thermal network itself can be used in combination with suitable control strategies to provide flexibility on the demand side (Vandermeulen et al., 2018). In the following, the use of renewable energies for the central heat and cold supply and also the power supply of bidirectional low- temperature networks is discussed in more detail on the basis of the simulation studies discussed above.

Bidirectional low-temperature networks offer numerous options for integrating renewable energies due to their temperature levels as well as the coupling of the heating, cooling and electricity sectors. Using the base case as example it can be seen from the temperature and power profiles of the central supply in Figure 5 and Figure 6 that in winter heat sources with a temperature above $20^{\circ} \mathrm{C}$ and in summer heat sinks with temperatures below $32^{\circ} \mathrm{C}$ can be used to balance the energy consumption of the network and to ensure the defined limits of the network temperatures. This enables a wide use of renewable energy technologies. The use of central waste heat (e.g. from data centers or industrial cooling processes), the operation of central heat pumps, the use of geothermal as well as solar thermal energy are suitable for the supply of heat. In combination with heat pumps, heat sources at lower temperature levels like ground-water or waste heat can also be integrated. The investigation of the integration of additional consumers has shown that the use of waste heat, which results in increased diversity indexes, leads to improved energy efficiency of the system. Geothermal energy, free cooling and compression chillers in combination with technologies for renewable power generation are examples of possible technologies for cold supply of the bidirectional lowtemperature network. The electricity demand resulting from the operation of heat pumps and chillers also enables the use of renewable electricity from PVsystems and wind turbines. Another efficient way of providing heat, which also provides the electricity needed to operate the bidirectional low-temperature network, is to use combined heat and power (CHP) units. During the winter, CHPs can feed heat into the network and at the same time supply the heat pumps and chillers with electricity. In combination with a central absorption chiller, the heat from the CHP unit can be used in summer to provide cold. Furthermore, the investigation of different temperature levels has shown that the network temperatures can be adapted to local renewable energy resources like river water, waste water of waste heat from industrial processes. Due to the thermal capacity of the bidirectional network, fluctuating energies can be fed in as long as the temperature limits are not reached. The integration of energy storages also provide improved conditions for the use of fluctuating renewable energy resources like solar thermal energy or electricity from $\mathrm{PV}$-systems and wind turbines. Short-term fluctuating energy sources can be utilized through the use of buffer storages, seasonal energy sources through the use of seasonal storage facilities.

\section{Conclusion and limitations}

Bidirectional low-temperature networks are an promising concept to provide heat and cold for city 
districts. These networks are characterized in particular by low temperatures and the simultaneous use for heating and cooling using decentralized heat pumps and chillers. The network allows energy balancing between buildings, thus enabling the connected buildings to supply each other with heat and cold which increases the energy efficiency of the system. In order to investigate the complex hydraulic behavior of bidirectional low-temperature networks and various system configurations, a dynamic simulation model of an existing district was developed and used in this paper. The results of the simulation studies show that many system configurations influence the operation and the energy demands of bidirectional lowtemperature networks. By modifying the network temperatures, the heating, cooling and electricity demands of the bidirectional network can be adjusted within certain limits. In addition, the impact of the demand structure on the energetic efficiency of the network was assessed by demonstrating the influence of additional, time-adjusted consumers. Through increased energy balancing, the additional heating and cooling demands could even lead to a reduction in the central heat and cold supply required to ensure temperature limits. Furthermore, the simulation results show that the integration of seasonal storage systems enable to completely replace the central cold supply in the example district. Through specific planning in accordance with the respective local renewable energy resources, bidirectional low-temperature networks offer various possibilities for the integration of renewable energies and show great potential for efficient and low emission heat and cold supply of city districts. However, the energy efficiency of bidirectional low-temperature networks strongly depends on the composition of the energy demands and the temperatures required by end users. Energy can only be shifted between buildings and thus recycled if heating and cooling demands occur simultaneously. Moreover, the required flow temperatures of the end users affect the COPs of the heat pumps and chillers and thus the electricity consumption. Especially in existing districts with high requirements on the flow temperatures, this leads to increased electricity consumption for the heating and cooling supply. The paper shows that dynamic thermo-hydraulic simulation models can make an important contribution to planning and operational control of bidirectional networks. For this reason, the existing simulation model will be extended in future research to include systems for central heat and cold supply. In addition, the integration of renewable energies through the use of the network's thermal inertia will be examined more in detail in the future.

\section{Acknowledgment}

This project has received funding from the European Unions Horizon 2020 research and innovation pro- gramme under grant agreement No 731289.

\section{References}

ectogrid $^{T M}$ (2019). ectogrid ${ }^{T M}$ powered by e.on, http://ectogrid.com/, 04.01.2019.

Florides, G. and S. Kalogirou (2005). Annual ground temperature measurements at various depths. In 8th REHVA World Congress, Clima, Lausanne, Switzerland.

Lang, W., T. Auer, K. M. Heissler, J. Metz, and I. Nemeth (2018). Potenziale von Niedrigtemperaturnetzen zur Steigerung des Anteils erneuerbarer Energien in Quartieren. Abschlussbericht. Fraunhofer IRB Verlag.

Lund, H., S. Werner, R. Wiltshire, S. Svendsen, J. E. Thorsen, F. Hvelplund, and B. V. Mathiesen (2014). 4th Generation District Heating (4GDH): Integrating smart thermal grids into future sustainable energy systems. Energy 68, 1-11.

Müller, D., M. Lauster, A. Constantin, M. Fuchs, and P. Remmen (2016). AixLib-An Open-Source Modelica Library within the IEA-EBC Annex 60 Framework. In BauSIM 2016, pp. 3-9.

Pass, R. Z., M. Wetter, and M. A. Piette (2018). A thermodynamic analysis of a novel bidirectional district heating and cooling network. Energy 144 , 20-30.

Sulzer, M. and D. Hangartner (2014). Kalte Fernwärme (Anergienetze)-Grundlagen/Thesenpapier. Lucerne: Lucerne University of Applied Sciences Switzerland.

The European Comission (2015). Fifth generation, low temperature, high exergy district heating and cooling networks.

van der Heijde, B., M. Fuchs, C. R. Tugores, G. Schweiger, K. Sartor, D. Basciotti, D. Müller, C. Nytsch-Geusen, M. Wetter, and L. Helsen (2017). Dynamic equation-based thermo-hydraulic pipe model for district heating and cooling systems. Energy Conversion and Management 151, 158 169.

Vandermeulen, A., B. van der Heijde, and L. Helsen (2018). Controlling district heating and cooling networks to unlock flexibility: A review. Energy 151, $103-115$.

Wetter, M., M. Fuchs, P. Grozman, L. Helsen, F. Jorissen, D. Müller, C. Nytsch-Geusen, D. Picard, P. Sahlin, and M. Thorade (2015). IEA EBC annex 60 modelica library-an international collaboration to develop a free open-source model library for buildings and community energy systems. In Proceedings of building simulation 2015. 